\title{
DIREKTE KONTAKTIERUNG DES ENZYMS (PQQ)-GDH UND ELEKTRODEN MITHILFE VON POLYMERMODIFIZIERTEN NANORÖHREN FÜR DIE ANWENDUNG IN BIOBRENNSTOFFZELLEN
}

\author{
I. Schubart, G. Göbel, F. Lisdat
}

\section{Zusammenfassung}

In dieser Studie präsentieren wir eine Enzymelektrode, bei der ein direkter Elektronentransfer (DET) zwischen der Pyrrolochinolinchinon-abhängigen Glukosedehydrogenase (PQQ)-GDH und einer Elektrode realisiert werden konnte. Hierfür wird eine Goldelektrode mit mehrwandigen Kohlenstoffnanoröhren [engl. multi-walled carbon nanotubes (MWCNT)] modifiziert, anschließend mit einem Copolymer aus Anilinderivaten überzogen und dann die (PQQ)-GDH (Acinetobacter calcoaceticus) kovalent immobilisiert. Die gepulste Polymersynthese wird hinsichtlich der Effektivität der bioelektrokatalytischen Umsetzung von Glukose optimiert. Die Glukoseoxidation startet bei einem Potential von $-0,1 \mathrm{~V}$ vs. $\mathrm{Ag} / \mathrm{AgCl}(1 \mathrm{M} \mathrm{KCl})$ und Stromdichten von bis zu $500 \mu \mathrm{A} / \mathrm{cm}^{2}(+0,1 \mathrm{~V})$ können erreicht werden. Der Messbereich für Glukose liegt bei 0,1-5 mM (+0,1 V vs. $\mathrm{Ag} / \mathrm{AgCl}$ ). Der dynamische Bereich ist bei höherem Potential auf bis zu $100 \mathrm{mM}(+0,4 \mathrm{~V}$ vs $\mathrm{Ag} / \mathrm{AgCl})$ erweitert.

Die Elektrode wird als Anode in einer Biobrennstoffzelle (BBZ) mit einer Bilirubinoxidase-modifizierten MWCNT/Gold-Kathode eingesetzt. Beide Elektroden basieren auf einem DET. Das Zellpotential der BBZ beträgt $680 \pm 20 \mathrm{mV}$ und sie erreicht eine maximale Leistungsdichte von $65 \mu \mathrm{W} / \mathrm{cm}^{2}$ (bei einer Zellspannung von $350 \mathrm{mV}$ ).

\section{Abstract}

In this study we present a (PQQ)-GDH electrode with direct electron transfer (DET) between the enzyme and electrode. Soluble pyrroloquinoline-quinone dependent glucose dehydrogenase from Acinetobacter calcoaceticus is covalently bound to an electropolymerized polyaniline copolymer film on a multi-walled carbon nanotube modified gold electrode. The pulsed electropolymerization of 2-methoxyaniline-5-sulfonic acid and $\mathrm{m}$-aminobenzoic acid is optimized with respect to the efficiency of the bioelectrocatalytic conversion of glucose. The glucose oxidation starts at $-0.1 \mathrm{~V}$ vs. $\mathrm{Ag} / \mathrm{AgCl}$ and current densities up to $500 \mu \mathrm{A} / \mathrm{cm}^{2}$ at a rather low potential can be achieved. The glucose sensitivity reaches from $0.1 \mathrm{mM}$ to $5 \mathrm{mM}$ at $+0.1 \mathrm{~V}$ vs. $\mathrm{Ag} / \mathrm{AgCl}$. The dynamic range is extended to $100 \mathrm{mM}$ at $+0.4 \mathrm{~V} \mathrm{vs}$. $\mathrm{Ag} / \mathrm{AgCl}$. The electron transfer mechanism and buffer effects are investigated.

The developed enzyme electrode is examined for bioenergetic application by assembling of a membrane-less biofuel cell. For the cathode a bilirubin oxidase based MWCNT modified gold electrode with DET is used. The biofuel cell has a cell potential of $680 \pm 20 \mathrm{mV}$ and a maximum power density of up to $65 \mu \mathrm{W} / \mathrm{cm}^{2}$ (with a cell potential of $350 \mathrm{mV}$ ).

\section{»I. EINLEITUNG}

Derzeit werden enzymatische Biobrennstoffzellen (EBBZ) intensiv auf ihre Einsatzmöglichkeit als Stromquelle in implantierbaren Geräten wie z. B. Herzschrittmacher oder Insulinpumpen untersucht (Barton et al. 2004). Hier werden Enzyme für die Oxidation von energiereichen Substraten wie z. B. Glukose, Fruktose oder Laktose zur Energiegewinnung genutzt. Der Vorteil der Enzyme liegt darin, dass sie unter physiologischen Bedingungen arbeiten und somit relativ leicht zu handhaben sind. Dagegen bieten die derzeit erreichten Leistungsdichten und die Stabilität noch Potential für weitere Verbesserungen.
Für den Aufbau solcher Biobrennstoffzellen (BBZ) werden unterschiedliche Oxidoreduktasen untersucht. Auf Kathodenseite werden hauptsächlich Multi-Kupfer-Enzyme wie die Bilirubinoxidase (BOD) (Schubert et al. 2009; Yehezkeli et al. 2011) oder die Laccase (Li et al. 2008; Zebda et al. 2011) eingesetzt. Anodenseitig werden Glukoseoxidase (GOD) sowie bevorzugt verschiedene Dehydrogenasen wie z. B. Nicotinsäureamid-Adenin-Dinukleotid (NAD)- oder Pyrrolochinolinchinon (PQQ)-abhängige Glukosedehydrogenasen (GDH) (Wen et al. 2011; Miyake et al. 2009; Gao et al. 2007; Yuhashi et al. 2005, Tanne et al. 2010), Alkoholdehydrogenase (ADH) (Topcagic and Minteer 2006) oder
Fruktosedehydrogenase (FDH) (Murata et al. 2009) verwendet.

Aufgrund ihrer Unempfindlichkeit gegenüber Sauerstoff und ihrer hohen katalytischen Aktivität werden solche Dehydrogenasen für den Aufbau von membranfreien BBZ verwendet. Um eine effiziente Kommunikation des Enzyms mit der Elektrode zu gewährleisten, können grundsätzlich zwei verschiedene Ansätze verfolgt werden. Einerseits kann der elektrische Kontakt mittels eines ShuttleMoleküls als mediatorvermittelter Elektronentransferprozess [engl. mediated electron transfer (MET)] realisiert werden. Andererseits kann das Enzym auch direkt durch Elektronentunneling 
[engl. direct electron transfer (DET)] kontaktiert werden. Im Falle eines MET wird anodenseitig ein Mediator mit einem Redoxpotential über dem des Enzyms benötigt, um einen effizienten Elektronentransfer zu erhalten. Das Redoxpotential des Mediators hat jedoch auch einen Einfluss auf das resultierende Zellpotential und somit auf die Leistungsdichte der BBZ. Wird hingegen ein direkter Elektronentransfer etabliert, kann das aktive Zentrum direkt mit der Elektrode interagieren. Der DET wird zumeist durch eine Funktionalisierung der Elektrodenoberfläche z. B. mit Kohlenstoffmaterialien realisiert (Schubert et al. 2009; Ivnitski et al. 2007; Weigel et al. 2007).

Für das Enzym (PQQ)-GDH werden unterschiedliche Strategien zur EnzymElektroden-Kontaktierung verfolgt. In den meisten Fällen wird der Kontakt mittels eines Mediators hergestellt. Beispiele hierfür sind unter anderem Ferrocenderivate (Razumiene et al. 2000; Laurinavicius et al. 2004), PQQ (Jin et al. 1998, Tanne et al. 2010), $\mathrm{N}$-methylphenanzonium (Malinauskas et al. 2004), 1-methoxy-5-methylphenazinium methyl sulfat(Igarashietal.2004), oder Polymere mit Osmiumkomplexen (Habermüller et al. 2003).

\section{» II. MATERIAL UND METHODEN}

Zitronensäure, $\mathrm{CaCl}_{2}$ sind von der Carl Roth $\mathrm{GmbH}+$ Co KG (D); EDC, Glukose, KHCF(II), KHCF(III), NHS und SulfoGMBS von der Sigma Aldrich Chemie $\mathrm{GmbH}$ (D). Ethanol, Natriumacetat, $\mathrm{KCl}$ sind von der NeoLab $\mathrm{GmbH}$ (D). Die Apo-(PQQ)-GDH ist eine freundliche Überlassung der Roche Diagnostics $\mathrm{GmbH}$ (D). PQQ kommt von der Wako Chemicals $\mathrm{GmbH}$ (D) und MES von der AppliChem $\mathrm{GmbH}$ (D). Alle wässrigen Lösungen werden mit gereinigtem Wasser aus dem „ultra clear direct“-System von SG Water (D) hergestellt.

\section{Elektrodenpräparation}

Die MWCNTs werden in $5 \mathrm{mM}$ CitratPhosphat-Puffer (CiP, pH 7) in einer Konzentration von $10 \mathrm{mg} / \mathrm{ml}$ suspendiert. Nach 10 min werden $50 \mu$ entnommen, mit $100 \mu \mathrm{l} 5 \mathrm{mM} \mathrm{CiP} \mathrm{pH} 7$ verdünnt und anschließend mit einem Ultraschallhomogenisator (SONOPULS Mini 20) behandelt.
Für die Rekonstitution der PQQ-GDH wird eine Lösung von $1 \mathrm{mg} / \mathrm{ml}$ ApoEnzym und $20 \mu \mathrm{M} P Q Q$ in $50 \mathrm{mM}$ MES-Puffer $\mathrm{pH} 6,5+1 \mathrm{mM} \mathrm{CaCl}_{2}$ hergestellt und für $3 \mathrm{~h}$ lichtgeschützt und bei Raumtemperatur inkubiert. Danach wird die Lösung aliquotiert und eingefroren.

Die Goldelektroden (BASi MF-2014, USA $A=2,01 \mathrm{~mm}^{2}$ oder CHI101, $\mathrm{NI} . \quad A=3,12 \mathrm{~mm}^{2}$ ) werden mit Wasser und Schleifpapier der Korngröße 1200, 2500 und 3000 (Dieter Schmid Feine Werkzeuge $\mathrm{GmbH}$ ) für jeweils $20 \mathrm{~s}$ poliert und dazwischen mit Wasser gespült. Nach dem letzten Schleifvorgang wird zusätzlich mit Ethanol (99\%) gespült. Auf die gereinigten Elektroden werden $2 \times 4 \mu$ lder behandelten MWCNT-Suspension gegeben und getrocknet.

Für die Elektropolymerisation werden Lösungen mit einer Monomer-Konzentration von insgesamt $0,1 \mathrm{M}$ in $1 \mathrm{M}$ Schwefelsäure hergestellt. Mittels der Methode „multiple pulse amperometry“ des PGSTAT12 (AutoLab, Software: GPES 4.9) erfolgt die Elektropolymerisation. Nach der Polymerisation wird der Polymerfilm in $1 \mathrm{M}$ Schwefelsäure zyklovoltammetrisch untersucht.

Die Enzymimmobilisierung erfolg kovalent über NHS/EDC-Chemie. Dazu wird die Elektrode für $15 \mathrm{~min}$ in 25 mM NHS und 100 mM EDC inkubiert. Die Immobilisierung des Enzyms erfolgt durch einstündige Inkubation von $20 \mu \mathrm{l}$ PQQ-GDH (1 mg/ml). Im Anschluss wird $3 x$ mit 50 mM MES-Puffer $(\mathrm{pH} 6,5+1 \mathrm{mM} \mathrm{CaCl}$ ) gespült. Die Elektroden werden in $50 \mathrm{mM}$ MESPuffer $\left(\mathrm{pH} \mathrm{6,5+1} \mathrm{mM} \mathrm{CaCl}_{2}\right)$ bei $4{ }^{\circ} \mathrm{C}$ gelagert. Im Falle der BOD wird eine bereits publizierte Prozedur mit einem heterobifunktionellen Reagens verwendet (Schubert et al. 2009). Der Waschschritt wird mit 5 mM CiP pH 7 durchgeführt. Die Elektroden werden in $5 \mathrm{mM}$ CiP $\mathrm{pH} 7$ bei $4^{\circ} \mathrm{C}$ gelagert.

\section{Elektrochemische Messungen}

Die voltammetrischen Messungen erfolgen mit einem PGSTAT12 (Autolab). Die Scanrate beträgt, wenn nicht anders erwähnt, $100 \mathrm{mV} / \mathrm{s}$. Als Referenzelektrode dient eine $\mathrm{Ag} / \mathrm{AgCl}, 1 \mathrm{M} \mathrm{KCl}$ (Fa. Microelectronics Inc. USA) Elektrode. Die Gegenelektrode besteht aus einem spiralförmigen Platindraht. Die Messzelle ist eine Eigenanfertigung der AG Biosystemtechnik und hat ein Volumen von $1 \mathrm{ml}$. Die Auswertung der voltammetrischen Messungen erfolgt mit der Software GPES 4.9 (Eco Chemie B. V., Niederlande).

Die OCP- und die galvanodynamischen Messungen werden an dem Gerät Reference 600 (Gamry Instruments, USA) durchgeführt. Für die Bestimmung der Leistung der Biobrennstoffzelle wird die Enzymanode an den Anschluss für die Arbeitselektrode und die Enzymkathode an den Anschluss der Gegenelektrode angeschlossen. Sowohl die Messungen des OCP als auch die galvanodynamischen Messungen werden in 5 mM MES-Puffer pH 6,5 + $1 \mathrm{mM} \mathrm{CaCl}_{2}$ + 20 mM Glucose durchgeführt. Soweit nicht anders erwähnt werden immer Dreifachbestimmungen durchgeführt.

\section{» III.ERGEBNISSE UND DISKUSSION}

Das Ziel dieser Arbeit ist die Entwicklung einer Proteinelektrode, die auf einem direkten Elektronentransfer zwischen der (PQQ)-GDH und einer MWCNT-modifizierten Goldelektrode basiert und in einer Biobrennstoffzelle als Anode eingesetzt werden kann. Die Kohlenstoffnanoröhren sollen hierbei die aktive Oberfläche der Elektrode erhöhen und bilden zudem eine ideale Basis für eine Elektroden-Enzym-Interaktion. Wie bereits in einer vorangegangenen Studie gezeigt, eignen sich CNTs mit einer zusätzlichen Zwischenschicht aus PQQMolekülen als Mediator, um die (PQQ)$\mathrm{GDH}$ elektrochemisch anzusprechen (Tanne et al. 2010). Um diese Interaktion zu verbessern und auf einen Mediator zu verzichten, wird eine neue Modifizierung entwickelt. Diese besteht aus einem Polyanilinfilm und wird durch Elektropolymerisation direkt auf der MWCNT-Oberfläche synthetisiert. Der Einfluss der Polymerisationsparameter auf die Filmeigenschaften und die damit verbundene Enzym-Elektroden-Interaktion wird analysiert. Weiterhin werden der Elektronentransfermechanismus, das Elektrodenverhalten in Anwesenheit von Glukose sowie der Einfluss der Lösungszusammensetzung untersucht. Die Elektrode wird abschließend mit einer bereits etablierten BOD-Kathode ähnlichen Aufbaus (Schubert et al. 2009) in einer Biobrennstoffzelle untersucht und ihre Leistungsparameter bestimmt. 


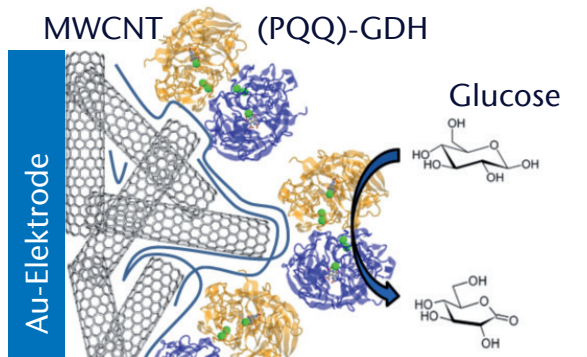

Gluconolacton

Abb. 1) Schematische Darstellung des Aufbaus der Enzymelektrode

\section{Polymersynthese}

Eine schematische Darstellung der entwickelten Elektrode ist mit Abbildung 1 gegeben. Die Grafik zeigt die MWCNTmodifizierte Goldelektrode mit einem Polymerfilm zur elektrochemischen Kontaktierung der Enzyme. Bei diesem Polymerfilm handelt es sich um Copolymere verschiedener Anilinderivate. Durch die Kombination verschiedener Monomere ist es möglich, die Eigenschaften des Polymers bereits vor der Polymerisation in einer gewünschten Richtung zu beeinflussen, wodurch eine nachträgliche Modifizierung unnötig wird. Untersucht werden die Monomere meta-Anilinsulfonsäure (ABS), 2-Methoxyanilin-4-sulfonsäure (MASA), meta-Aminobenzoesäure (ABA) und 2-Methoxyanilin (MA). Die beiden ersten tragen eine Sulfonsäuregruppe, wodurch die Leitfähigkeit des Polymers auch bei physiologischen Bedingungen positiv beeinflusst wird. ABA dient mit der Carboxylgruppe zur kovalenten Kopplung des Enzyms und MA kann genutzt werden, um das Makromolekül weniger hydrophil zu gestalten.

Die Elektropolymerisation (EP) erfolgt durch eine potentiostatische Pulsmethode. Da hier eine Reihe an Parametern den resultierenden Polymerfilm beeinflussen können, werden diese untersucht und hier kurz zusammengefasst. Die Abbildung 2 zeigt rasterelektronenmikroskopische Aufnahmen modifizierter Elektroden: a) Gold/MWCNT und b) Gold/MWCNT/Polymer. Die veränderte Oberflächenstruktur durch den synthetisierten Copolymerfilm auf den MWCNTs ist deutlich zu erkennen.

Um die Güte der Kontaktierung des Proteins mit der Elektrode einschätzen zu können, werden alle präparierten Elektroden nach der EP mit der (PQQ)$\mathrm{GDH}$ gekoppelt. Anschließend werden LSV-Messungen in An- und Abwesenheit von Glukose durchgeführt und die resultierenden Ströme als Vergleichsgröße herangezogen. Mit Ausnahme von Polymerfilmen mit MA in der Monomerlösung ist mit allen synthetisierten Polymerfilmen die elektrochemische Kontaktierung des Enzyms möglich.

Es zeigt sich jedoch, dass das Pulspotential über 0,4 V liegen muss, um die Monomere zu polymerisieren. Ein Optimum wird bei Potentialen zwischen 0,6 V und $0,8 \vee$ festgestellt. Die Anzahl der Pulse und somit die Länge der Polymerisation hat ebenfalls einen großen Einfluss. So kann der katalytische Strom bei 300 s Polymerisationszeit gegenüber $50 \mathrm{~s}$ von ca. $50 \mu \mathrm{A} / \mathrm{cm}^{2}$ auf bis $\mathrm{zu}$ $400 \mu \mathrm{A} / \mathrm{cm}^{2}$ (5 mM Glucose bei 0,1 V vs. $\mathrm{Ag} / \mathrm{AgCl}$ ) erhöht werden. CV-Messungen deuten hier auf eine vergrößerte Oberfläche hin. Die Steigerung ist jedoch limitiert und endet bei $300 \mathrm{~s}$. Auch die Monomerzusammensetzung sowohl in der Auswahl der Monomere als auch im Verhältnis zweier Monomere hat einen deutlichen Einfluss auf den resultierenden Strom. Für die Untersuchungen werden folgende Parameter der EP als optimal befunden: Pulspotential $=0,6 \mathrm{~V}$, Länge der Pulses $0,8 \mathrm{~s}$;
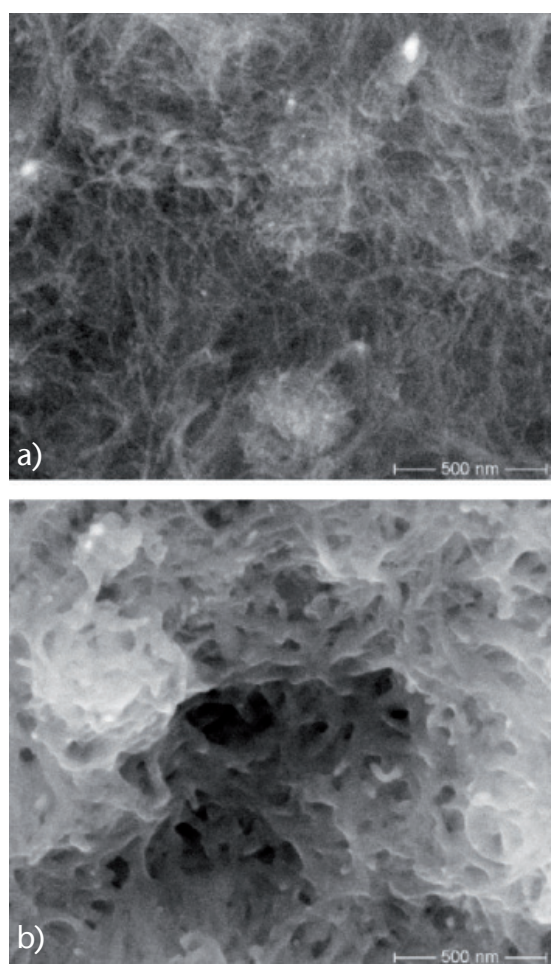

Abb. 2) REM-Aufnahmen der modifizierten Elektroden a) Gold/MWCNT $(15 \mathrm{kV})$, b) Gold/MWCNT/Polymer (10 kV)
Ruhepotential 0 V, Länge des Ruhepotentials $0,1 \mathrm{~s}$; Dauer der Polymerisation=300 s; Monomere: MASA + ABA im Verhältnis 4:1 in der Monomerlösung.

\section{Charakterisierung des}

Elektronentransfermechanismus

Um den Mechanismus des Elektronentransfers zwischen dem Enzym und der Elektrode genauer aufzuklären, werden verschiedene Experimente durchgeführt. Zunächst soll überprüft werden, ob dem katalytischen Strom tatsächlich die enzymatische Oxidation der Glukose zugrunde liegt. Dazu wird das nichtaktive Apoenzym der GDH an die Oberfläche gekoppelt. Durch LSV-Messungen in An- und Abwesenheit von Glukose kann gezeigt werden, dass diese Elektrode keinen katalytischen Strom generiert. Der zuvor detektierte Strom mit dem Holoenzym basiert demnach nur auf der katalytischen Aktivität der (PQQ)-GDH und nicht auf Umsatzreaktionen direkt an der modifizierten Elektrode.

Im Folgenden soll nun der Mechanismus näher untersucht werden. Aus einer früheren Studie ist bekannt, dass freies PQQ als Mediator zwischen dem Enzym und der Elektrode fungieren kann. Möglicherweise könnte PQQ aus dissoziierten Holoenzymmolekülen auch in der hier vorgestellten Elektrodenkonfiguration bei dem Elektronentransfer vermitteln. Aus diesem Grund wird zunächst das Verhalten von PQQ an der polymermodifizierten MWCNTElektrode untersucht. Zyklovoltammetrische Messungen zeigen schon bei sehr kleinen Vorschubgeschwindigkeiten relativ große Peakseparationen von $182 \pm 17 \mathrm{mV}$ (siehe Abb. 3a). Die heterogene Elektronentransfergeschwindigkeitskonstante zwischen dem PQQ und der modifizierten Elektrode kann bestimmt werden $\left(1,59 \cdot 10^{-3} \pm\right.$ $\left.0.14 \cdot 10^{-3} \mathrm{~cm} / \mathrm{s}\right)$ und spricht für einen langsamen Prozess.

In einem weiteren Versuch wird die komplette Enzymelektrode hergestellt und LSV-Messungen in An- und Abwesenheit von freiem PQQ in Lösung durchgeführt. Die Ergebnisse sind in Abbildung $3 b$ dargestellt. Die Kurve I repräsentiert hier die Grundlinie ohne Glukose und PQQ in Lösung. Kurve II zeigt den Stromverlauf in $10 \mathrm{mM}$ 
Glukoselösung. Die Kurven III bis V stellen den Verlauf bei sukzessivem Zusatz von freiem $P Q Q$ in der Glukoselösung dar. Bei geringen PQQ-Konzentrationen ist in der Stromantwort zunächst keine signifikante Änderung zu beobachten. Erst bei höheren PQQ-Konzentrationen steigt bei höheren Potentialen der katalytische Strom. Hieraus lässt sich ableiten, dass PQQ, wie vermutet, als Mediator fungieren kann, jedoch wird dazu eine höhere Konzentration benötigt und der Effekt tritt erst bei Potentialen über $150 \mathrm{mV}$ vs. Ag/AgCl ein. Für die polymer gebundene (PQQ)-GDH kann nach der Interpretation der durchgeführten Experimente der direkte Elektronentransfer als der dominierende Mechanismus identifiziert werden.

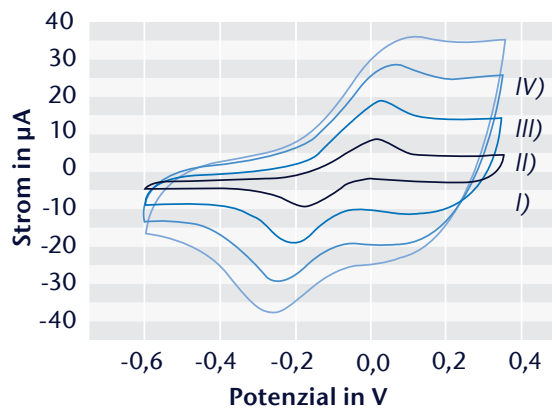

Abb. 3a) Cyclovoltammogramm einer poly $[A B A$ MASA]/MWCNT/Gold-Elektrode in $1 \mathrm{mM} P Q Q$ Lösung bei verschiedenen Scanraten I $5 \mathrm{mV} / \mathrm{s}$, II $20 \mathrm{mV} / \mathrm{s}$, III $40 \mathrm{mV} / \mathrm{s}$ und IV $60 \mathrm{mV} / \mathrm{s}$ (5 mM MES, pH 6,5 +1 mM $\left(\mathrm{CaCl}_{2}\right.$ )

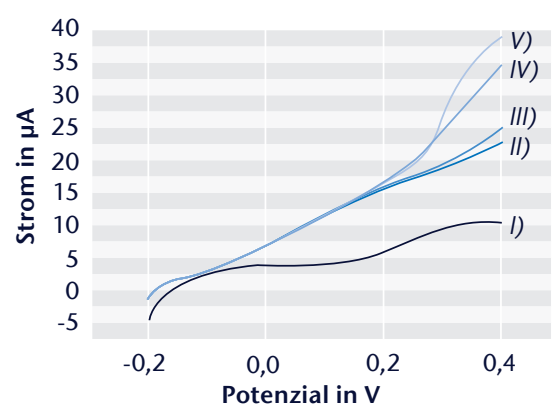

Abb. 3b) LSV einer (PQQ)-GDH/poly[ABA/MASA]/ MWCNT/Gold-Elektrode in Abhängigkeit von PQQ in Lösung: I ohne Glucose, II $10 \mathrm{mM}$ Glucose, III $10 \mathrm{mM}$ Glucose + $5 \mu \mathrm{M} P Q Q$, IV $10 \mathrm{mM}$ Glucose + $10 \mu \mathrm{M}$ PQQ, $\checkmark 10 \mathrm{mM}$ Glucose + $30 \mu \mathrm{M}$ PQQ $(10 \mathrm{mV} / \mathrm{s}, 5 \mathrm{mM}$ MES $\left.\mathrm{pH} 6,5+1 \mathrm{mM} \mathrm{CaCl}_{2}, \mathrm{Evs} . \mathrm{Ag} / \mathrm{AgCl}, 1 \mathrm{M} \mathrm{KCl}\right)$

\section{Elektrochemische Charakterisierung}

Die elektrochemische Charakterisierung der Enzymelektrode und potentiellen Anode in einer BBZ umfasst den Einfluss der lonenstärke, des $\mathrm{pH}$-Wertes sowie die Sensitivität gegenüber Glukose und die Stabilität. Abbildung 4a zeigt den Zusammenhang der Stromantwort in $1 \mathrm{mM}$ Glukoselösung bei $100 \mathrm{mV}$ vs.
$\mathrm{Ag} / \mathrm{AgCl}$ und unterschiedlichen $\mathrm{pH}$ Werten mit einem Maximum bei pH 6,5. Auch bei geringen Abweichungen von diesem Optimum können noch Stromdichten von $90 \%$ des Maximalwertes erreicht werden. Bei $\mathrm{pH}$-Werten $\leq 5,5$ geht die Stromantwort gegen Null. Wird der $\mathrm{pH}$-Wert anschließend wieder erhöht, kann der resultierende Strom auch nicht wieder vergrößert werden. Diese Gegebenheit kann durch eine irreversible Änderung in der Protein-Polymer-Konnektivität erklärt werden, da das aktive Zentrum des Enzyms auch unter sauren Bedingungen funktionsfähig ist.

Der Einfluss der lonenstärke auf das System wird in zwei Experimenten untersucht. Zum einem wird die Pufferkomponente 2-(-N-morpholino) ethansulfonsäure (MES) in einem Konzentrationsbereich zwischen $1 \mathrm{mM}$ und $75 \mathrm{mM}$ variiert, zum anderen wird Kalzium in verschiedenen Konzentration der

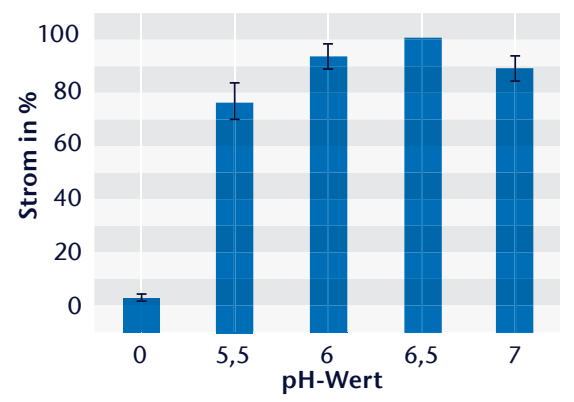

Abb. 4a) Stromantwort einer (PQQ)-GDH/poly[ABA/ MASA]/MWCNT/Gold-Elektrode in Anwesenheit von 1 $m M$ Glucose bei 0,1 V vs. Ag/AgCl, $1 \mathrm{M} \mathrm{KCl}$ in Abhängigkeit von $\mathrm{pH}$-Wert
Pufferlösung zugesetzt. Es zeigt sich, dass das katalytische System unabhängig von der MES-Konzentration arbeitet (Abb. 4b). Kalzium hingegen hat einen deutlichen Einfluss auf die Stromantwort (Abb. 4c). Pro Untereinheit der (PQQ)-GDH sind drei Kalziumionen gebunden. Sie sind sowohl für die koordinative Bindung der prosthetischen Gruppe als auch für die Assoziation der beiden Untereinheiten verantwortlich (Olsthoorn and Duine 1996; Oubrie et al. 1999). Wie bereits in früheren Studien berichtet, kann die Anwesenheit geringer Konzentrationen an $\mathrm{Ca}^{2+}$, das Signal beeinflussen (Tanne et al. 2010; jin et al. 1995). Ein Einfluss von $\mathrm{Ca}^{2+}$ auch bei höheren Konzentrationen, kann in dieser Studie belegt werden. Dieser ist wahrscheinlich auf eine bessere Enzyminteraktion mit der Oberfläche zurückzuführen.

Für die weitere Charakterisierung der Elektrode wird die Sensitivität gegenüber dem Substrat mit LSV-Messungen ermittelt. Wie in Abbildung 5 erkennbar, ist der katalytische Strom abhängig vom angelegten Potential. Bei geringem Potential von $0,1 \mathrm{~V}$ vs. $\mathrm{Ag} / \mathrm{AgCl}$ kann eine Inhibierung für Glukosekonzentrationen von $10 \mathrm{mM}$ beobachtet werden. Dieser Effekt ist auch für GDH in Lösung bekannt (Dokter et al. 1986). Bei 0,4 V vs. $\mathrm{Ag} / \mathrm{AgCl}$ hingegen wird bis zu $100 \mathrm{mM}$ Glukose keine Substratinhibition festgestellt. Diese Beobachtungen lassen darauf schließen, dass hier nicht nur

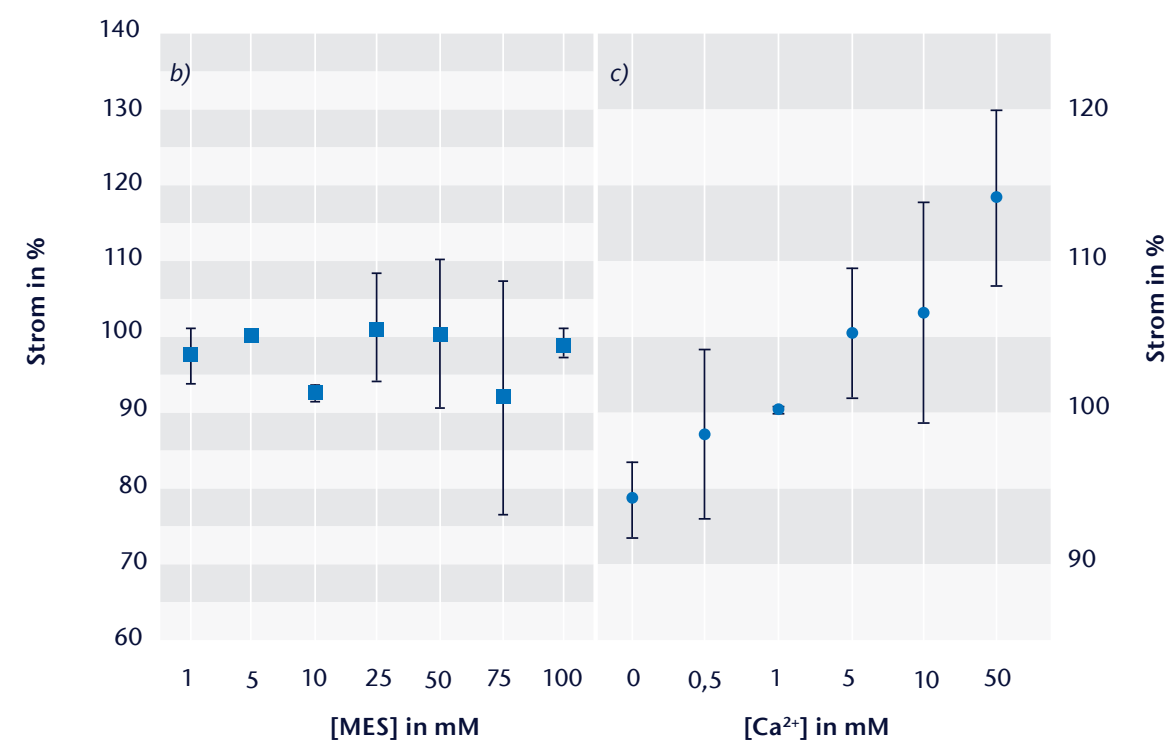

Abb. $4 b+c)$ Stromantwort einer (PQQ)-GDH/poly[ABA/MASA]/MWCNT/Gold-Elektrode in Anwesenheit von $1 \mathrm{mM}$ Glucose bei $0,1 \mathrm{Vvs}$. Ag/AgCl, $1 \mathrm{M} \mathrm{KCl}$ in Abhängigkeit von MES-Konzentration (b) und $\mathrm{CaCl}_{2}$-Konzentration (10 mV/s, $5 \mathrm{mM} \mathrm{MES} \mathrm{pH} \mathrm{6,5+1} \mathrm{mM} \mathrm{CaCl}_{2}$, Evs. Ag/AgCl, $1 \mathrm{M} \mathrm{KCl}$ ) (c) 


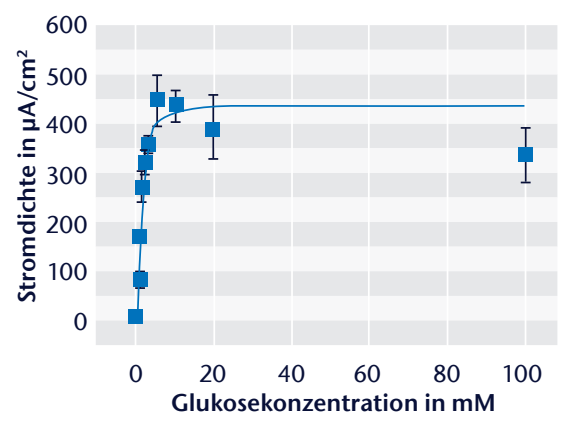

Abb. 5a) Glukosesensitivität einer (PQQ)-GDH/poly [ABA/MASA]/MWCNT/Gold-Elektrode bei 0,1 V

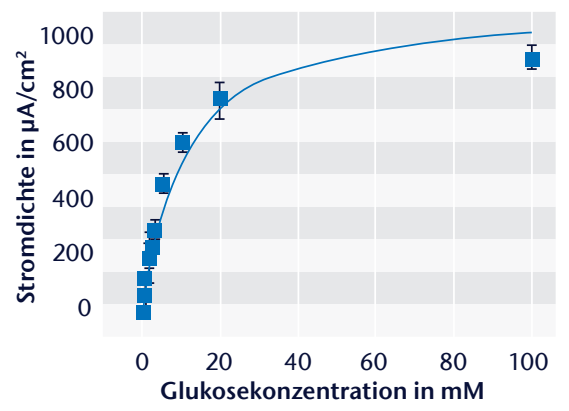

Abb. 5a) Glukosesensitivität einer (PQQ)-GDH/poly [ABA/MASA]/MWCNT/Gold-Elektrode bei 0,4 V vs. Ag/ $\mathrm{AgCl}, 1 \mathrm{M} \mathrm{KCl}(10 \mathrm{mV} / \mathrm{s}, 5 \mathrm{mM} \mathrm{MES} \mathrm{pH} 6,5+1 \mathrm{mM}$ $\left.\mathrm{CaCl}_{2}\right)$

die Enzymkinetik, sondern auch die Elektronentransferrate und die Orientierung der elektrokatalytischen Domäne zur Elektrode einen Einfluss haben. Offensichtlich kann das Enzym bei höherem Potential schneller regeneriert werden und somit mehr Glukose oxidiert werden. Solche Effekte unterschiedlicher Leistungsfähigkeiten werden auch in einer aktuellen Studie über GDH-Mutanten an Elektroden gefunden (Flexer et al. 2010).

Die apparente Michaelis-Menten-Konstante $\mathrm{K}_{\mathrm{M}}$, App , liegt für $0,1 \mathrm{~V}$ vs. $\mathrm{Ag} / \mathrm{AgCl}$ bei 2,0 mM Glukose und für 0,4 V vs. $\mathrm{Ag} / \mathrm{AgCl}$ bei $10,5 \mathrm{mM}$. Wird die Elektrode bei $4^{\circ} \mathrm{C}$ in $5 \mathrm{mM}$ MES Puffer $\mathrm{pH} 6,5$ und $50 \mathrm{mM} \mathrm{CaCl}_{2}$ gelagert, ist eine Abnahme der Stromantwort bereits nach einem Tag zu beobachten. Innerhalb der folgenden vier Tage stabilisiert sich das Signal und beträgt rund $50 \%$ des Ausgangswertes.

\section{Anwendung in einer}

\section{Biobrennstoffzelle}

Wie bereits beschrieben, ist bei dem Aufbau einer EBBZ mit (PQQ)-GDH keine Membran zwischen Anode und Kathode nötig. Die entwickelte Enzymelektrode wird in diesem Anwendungsbereich für die katalytische Umwandlung der chemischen Energie von Glukose in elektrische Energie eingesetzt.

Kathodenseitig wird BOD auf eine MWCNT-modifizierte Goldelektrode immobilisiert (Schubert et al. 2009). Beide Elektroden basieren auf einem direkten Elektronentransfer und erreichen Stromdichten im Bereich mehrerer hundert $\mu \mathrm{A} / \mathrm{cm}^{2}$. Damit die Brennstoffzelle optimal arbeitet, sollten die Halbzellenpotentiale der beiden Elektroden weit auseinander liegen, damit die Gesamtzellspannung möglichst groß ist. Das Ruhepotential der Anode liegt bei $-120 \pm$ $9 \mathrm{mV}$ vs. $\mathrm{Ag} / \mathrm{AgCl}(\mathrm{n}=4)$, das der Kathode bei $560 \pm 12 \mathrm{mV}$ vs. $\mathrm{Ag} / \mathrm{AgCl}(\mathrm{n}=4)$. Folglich beträgt das offene Zellpotential (OCP) $680 \pm 20 \mathrm{mV}(\mathrm{n}=4)$.

Zunächst wird die Stabilität der Zelle unter Last (durch periodisches Anlegen eines $500 \mathrm{k} \Omega$-Widerstands) analysiert. Durch erstmaliges Anlegen des Widerstands fällt das Zellpotential von $660 \mathrm{mV}$ auf $510 \mathrm{mV}$ ab. Nach $3 \mathrm{~h}$ periodischen Anlegens des $500 \mathrm{k} \Omega$-Widerstandes sinkt das OCP auf $431 \mathrm{mV}$. Bei vorangegangenen Studien mit einer MET basierten Anode betrug die Differenz zwischen dem Ausgangswert nach einmaliger Belastung $250 \mathrm{mV}$, in dieser Arbeit beträgt der Verlust lediglich $150 \mathrm{mV}$, wodurch belegt wird, dass der direkte Elektronentransfer die Leistung der Brennstoffzelle positiv beeinflusst. Nach $3 \mathrm{~h}$ beträgt die Differenz 207 mV und liegt damit immer noch deutlich unter der Vergleichsmessung und zeigt die deutlich verbesserte Stabilität des Systems durch Etablierung eines DET (Abb. 6a).

Weitere Charakteristika einer EBBZ sind die Spannung und die Leistungsdichte in Abhängigkeit der Stromdichte (siehe Abb. 6b). Die Leistungskurven werden durch galvanodynamische Messungen bestimmt. Sie zeigen eine maximale Leistungsdichte von $65 \mu \mathrm{W} / \mathrm{cm}^{2}$ bei einem Zellpotential von $350 \mathrm{mV}$. Bei geringen Stromdichten ist ein linearer Anstieg der Leistungsdichte zu beobachten. Wird das Maximum bei einer Stromdichte von $180 \mu \mathrm{A} / \mathrm{cm}^{2}$ erreicht, fällt die Leistungskurve mit höher werdender Stromdichte wieder deutlich ab. Die erreichte Stromdichte mit diesem System ist dreimal größer als bei der EBBZ mit MET auf der Anodenseite (Tanne et al. 2010) oder einer Biobrennstoffzelle, bei der eine (PQQ)-
GDH Mutante an eine Kohlenstoffpastenelektrode gebunden ist (Yuhashi et al. 2005). Sie ist vergleichbar mit denen von Li et al. (Li et al. 2008) erreichten Leistungsdichten, die SWCNT-modifizierte Kohlenstofffaser-Elektroden mit Methylengrün nutzten und (NAD+)$\mathrm{GDH}$ an der Anode und Laccase an der Kathode immobilisierten, sowie bei Gao et al. (Gao et al. 2007), welche Poly(Brilliantkresylblau) an SWCNT-modifizierten Glaskohlenstoffelektroden verwendeten. Die Literatur gibt aber auch einige Beispiele für höhere Leistungsdichten. So können mit dem Enzym Alkoholdehydrogenase, immobilisiert an einer Tetraalkylammoniumbromid/Nafion Membran, und BOD, eingebettet in einer $\mathrm{Ru}(\mathrm{bpy}){ }_{3}{ }^{2+} / \mathrm{Nafion}$ Membran, Werte von bis zu $460 \mu \mathrm{W} /$ $\mathrm{cm}^{2}$ erreicht werden (Topcagic and Minteer 2006). Ein anderes System arbeitet mit Fruktosedehydrogenase und ebenfalls BOD in einer dreidimensionalen Goldnanopartikel-modifizierten Goldelektrode und erreicht $660 \mu \mathrm{W} / \mathrm{cm}^{2}$ (Murata et al. 2009).

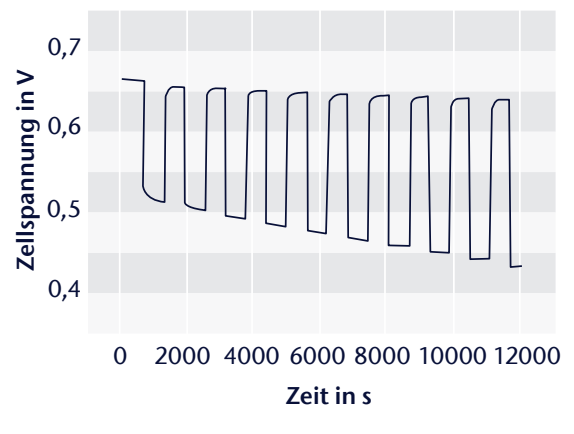

Abb. 6a) Stabilität des Zellpotentials mit und ohne Last von $500 \mathrm{k} \Omega$

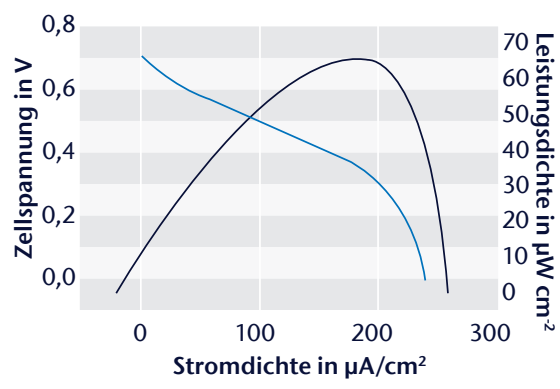

$A b b .6 b)$ Leistungskurven der assemblierten EBBZ mit $20 \mathrm{mM}$ Glucose (5 nA/s, $5 \mathrm{mM}$ MES pH 6,5 + $1 \mathrm{mM}$ $\left.\mathrm{CaCl}_{2}, \mathrm{Evs} . \mathrm{Ag} / \mathrm{AgCl}, 1 \mathrm{M} \mathrm{KCl}\right)$

\section{»IV.FAZIT}

Ziel dieser Arbeit war die Entwicklung einer Enzymelektrode auf Basis einer MWCNT modifizierten Goldelektrode, die mit einem Polymerfilm überzogen 
wird, um anschließend das Enzym (PQQ)-GDH kovalent zu koppeln und elektrisch anzusprechen. Um den Polymerfilm zu optimieren, wurden Parameter der Elektropolymerisation sowie unterschiedliche Monomere und deren Mischungsverhältnisse untersucht. Es zeigt sich, dass ein Copolymer aus 2-Methoxyanilin-4-sulfonsäure und meta-Aminobenzoesäure am besten geeignet ist. In Anwesenheit von $5 \mathrm{mM}$ Glucose können Stromdichten von bis zu $500 \mu \mathrm{A} / \mathrm{cm}^{2}$ generiert werden $(0,1 \mathrm{~V}$ vs. Ag/AgCl). Die katalytische Umsatzreaktion startet dabei schon bei $-0,1 \mathrm{~V}$ vs. $\mathrm{Ag} / \mathrm{AgCl}$ mit einem dynamischen Messbereich zwischen 0,1 $\mathrm{mM}$ und $10 \mathrm{mM}$ Glucose, der bei höheren Potentialen auf bis zu 100 mM Glucose erweitert ist. Weiterhin ist der Elektronentransfermechanismus in verschiedenen Experimenten analysiert worden und kann als direkter ET belegt werden. Gezeigt wurde zudem, dass die Elektrode in einem gewissen Bereich unabhängig von der Pufferkonzentration ist, Calciumionen hingegen die katalytische Reaktion positiv beeinflussen.

Abschließend wurde die Elektrode in einer enzymatischen Biobrennstoffzelle eingesetzt. Kathodenseitig wurde eine Bilirubinoxidase-Elektrode verwendet. In diesem membranlosen System, in dem sowohl Anode als auch Kathode auf MWCNT-modifizierten Goldelektroden mit direktem ET basieren, konnte eine relativ hohe Zellspannung von bis zu $700 \mathrm{mV}$ erreicht werden. Die maximale Leistungsdichte beträgt $65 \mu \mathrm{W} / \mathrm{cm}^{2}$.

\section{LITERATUR}

Barton, S. C., Gallaway, I., Atanassov, P. (2004): Enzymatic Biofuel Cells for Implantable and Microscale Devices. Chemical Review, 104, 4867-4886.

Dokter, P., Frank, J., Duine, J. A. (1986): Purification and characterization of quinoprotein glucose dehydrogenase from Acinetobacter calcoaceticus L. M . D . 79 . 41. Biochemical Journal, 239, 163-167.

Flexer, V., Brun, N., Backov, R., Mano, N. (2010): Designing highly efficient enzyme-based carbonaceous foams electrodes for biofuel cells. Energy \& Environmental Science, $3(9), 1302$.

Flexer, V., Durand, F., Tsujimura, S., Mano, N. (2011): Efficient Direct Electron Transfer of PQQ-glucose Dehydrogenase on Carbon Cryogel Electrodes at Neutral pH. Analytical Chemistry, 83 (14), 5721-5727.

Gao, F., Yan, Y., Su, L., Wang, L., Mao, L. (2007): An enzymatic glucose/O2 biofuel cell: Preparation, charac terization and performance in serum. Electrochemistry Communications, 9 (5), 989-996.

Göbel, G., Lisdat, F. (2008): Organic interlayers for oxygen reducing electrodes based on bilirubin oxidase and MWCNT modified gold. Electrochemistry Communications, 10 (11), Elsevier B.V., 1691-1694.

Habermüller, K., Reiter, S., Buck, H., Meier, T., Staepels, J., Schuhmann, W. (2003): Conducting Redoxpolymer-Based Reagentless Biosensors Using Modified PQQ-Dependent Glucose Dehydrogenase. Microchimica Acta, 143 (2-3), $113-121$

Igarashi, S., Okuda, J., Ikebukuro, K., Sode, K. (2004): Molecular engineering of $\mathrm{PQQGDH}$ and its applications. Archives of Biochemistry and Biophysics, 428 (1), 52-63.

Ivnitski, D., Atanassov, P., Apblett, C. (2007): Direct Bioelectrocatalysis of PQQ-Dependent Glucose Dehydrogenase. Electroanalysis, 19 (15).

Jin, W., Bier, F., Wollenberger, U., Scheller, F. (1995): Construction and characterization of a multi-layer enzyme electrode: Covalent binding of quinoprotein glucose dehydrogenase onto gold electrodes. Biosensors \& Bioelectronics, 10, 823-829.

Jin, W., Wollenberger, U., Scheller, F. W. (1998): PQQ as Redox Shuttle for Quinoprotein Glucose Dehydrogenase. Biologycal Chemistry, 379 (August/September) 1207-1211.

Laurinavicius, V., Razumiene, J., Ramanavicius, A., Ryabov, A. D. (2004): Wiring of PQQ-dehydrogenases. Biosensors $\&$ Bioelectronics, 20 (6), 1217-1222. [Online]. Available at: doi:10.1016/j.bios.2004.05.012 [Accessed: 24 July 2011]

Li, X., Zhou, H., Yu, P., Su, L., Ohsaka, T., Mao, L. (2008): A Miniature glucose/O2 biofuel cell with single-walled carbon nanotubes-modified carbon fiber microelectrode as the substrate. Electrochemistry Communications, 10 (6), 851-854.

Malinauskas, A., Kuzmarskyt, J., Meskys, R., Ramanavicius, A. (2004): Bioelectrochemical sensor based on PQQ-dependent glucose dehydrogenase. Sensors and Actuators B, 100 (3), 387-394.

Miyake, T, Oike, M., Yoshino, S., Yatagawa, Y., Haneda, K, Kaji, H., Nishizawa, M. (2009): Biofuel cell anode: NAD+/ glucose dehydrogenase-coimmobilized ketjenblack electrode. Chemical Physics Letters, 480 (1-3), 123-126.

Murata, K., Kajiya, K., Nakamura, N., Ohno, H. (2009): Direct electrochemistry of bilirubin oxidase on threedimensional gold nanoparticle electrodes and its application in a biofuel cell. Energy \& Environmental Science, $2(12), 1280$.

Olsthoorn, A. J. J., Duine, J. A. (1996): Production , Characterization , and Reconstitution of Recombinant Quinoprotein Glucose Dehydrogenase Acinetobacter calcoaceticus. Archives of Biochemistry and Biophysics, 336 (1), 42-48.
Oubrie, A., Rozeboom, J., Kalk, K. H., Olsthoorn, A. J. J., Duine, J. A., Dijkstra, B. W. (1999): Structure and mechanism of soluble quinoprotein glucose dehydrogenase. The EMBO Journal, 18 (19), 5187-5194.

Razumiene, J., Meškys, R., Gureviciene, V., Laurinavicius, V., Reshetova, M. D., Ryabov, A. D. (2000): 4-Ferrocenylphenol as an electron transfer mediator in PQQ-dependent alcohol and glucose dehydrogenasecatalyzed reactions. Electrochemistry Communications, 2 (5), 307-311.

Razumiene, J., Vilkanauskyte, A., Gureviciene, V. (2006): Direct electron transfer between $\mathrm{PQQ}$ dependent glucose dehydrogenases and carbon electrodes: An approach for electrochemical biosensors. Electrochimica Acta, 51, 5150-5156

Sarauli, D., Riedel, M., Wettstein, C., Hahn, R., Stiba, K., Wollenberger, U., Leimkuhler, S., Schmuki, P., Lisdat, F. (2012): Semimetallic TiO2 nanotubes: new interfaces for bioelectrochemical enzymatic catalysis. J. Mater. Chem. 22 (11), The Royal Society of Chemistry, 4615-4618.

Schubert, K., Goebel, G., Lisdat, F. (2009): Bilirubin oxidase bound to multi-walled carbon nanotube-modified gold. Electrochimica Acta, 54 (11), 3033-3038.

Tanne, C., Göbel, G. and Lisdat, F. (2010): Development of a (PQQ)-GDH-anode based on MWCNT-modified gold and its application in a glucose/O2-biofuel cell. Biosensors \& Bioelectronics, 26 (2), Elsevier B.V., 530-535.

Topcagic, S., Minteer, S. D. (2006): Development of a membraneless ethanol/oxygen biofuel cell. Electrochimica Acta, 51 (11), 2168-2172.

Weigel, M. C., Tritscher, E., Lisdat, F. (2007): Direct electrochemical conversion of bilirubin oxidase at carbon nanotube-modified glassy carbon electrodes. Electrochemistry Communications, 9 (4), 689-693.

Wen, D., Xu, X., Dong, S. (2011): A single-walled carbon nanohorn-based miniature glucose/air biofuel cell for harvesting energy from soft drinks. Energy \& Environmental Science, 4 (4), 1358.

Yehezkeli, O., Tel-Vered, R., Raichlin, S., Willner, I. (2011): Nano-engineered flavin-dependent glucose dehydrogenase/gold nanoparticle-modified electrodes for glucose sensing and biofuel cell applications. ACS nano, 5 (3), 2385-2391.

Yuhashi, N., Tomiyama, M., Okuda, J., Igarashi, S., Ikebukuro, K., Sode, K. (2005): Development of a novel glucose enzyme fuel cell system employing protein engineered $\mathrm{PQQ}$ glucose dehydrogenase. Biosensors \& Bioelectronics, 20 (10), 2145-2150.

Zayats, M., Katz, E., Baron, R., Willner, I. (2005): Reconstitution of apo-glucose dehydrogenase on pyrroloquinoline quinone-functionalized au nanoparticles yields an electrically contacted biocatalyst. Journal of the American Chemical Society, 127 (35), 12400-12406.

Zebda, A., Gondran, C., Le Goff, A., Holzinger, M., Cinquin, P., Cosnier, S. (2011): Mediatorless highpower glucose biofuel cells based on compressed carbon nanotube-enzyme electrodes. Nature Communications, 2 (may), Nature Publishing Group, 370.

\section{AUTOREN}

Ivo Schubart, M.Sc.

Biosystemtechnik/Bioinformatik

Fachbereich Ingenieurwesen/Wirtschaftsingenieurwesen

TH Wildau [FH]

ivoschubart@aol.com

Dipl.-Biotechnologe Gero Göbel

Fachbereich Ingenieurwesen/Wirtschaftsingenieurwesen TH Wildau [FH] gero.goebel@th-wildau.de

Prof. Dr. Lisdat, Fred

Fachbereich Ingenieurwesen/Wirtschaftsingenieurwesen TH Wildau [FH]

fred.lisdat@th-wildau.de 